\title{
Research on musculoskeletal model of elbow joint for evaluating the feasibility of FES
}

\author{
Shengxin Wang ${ }^{*}$, Yongsheng Gao, Gangfeng Liu, Feiyun Xiao and Jie Zhao \\ State Key Laboratory of Robotics and System, School of Mechatronics Engineering, Harbin Institute \\ of Technology, No. 2 Yikuang Street, Nan Gang District, Harbin 150001, P.R. China
}

\begin{abstract}
Tremor usually occurs in a patient's upper limb with a roughly sinusoidal profile. Understanding the inner mechanism of the involuntary movement is fundamental to improving tremor suppression treatments. Therefore, the musculoskeletal model of the elbow joint was developed in this study. Initially, healthy subjects were selected to simulate tremor and the tremulous data was collected with the purpose of sparing patients from fatigue. With the recorded joint angle and surface EMG (sEMG), the model was calibrated to subjects by optimization approach. The activation derived from the electric pulse was employed to drive the tuned model and the model's output was compared with the angle predicted by the EMG-driven musculoskeletal model. The results demonstrated that the performance of the calibrated model was improved by a smaller normalized root mean square error and a higher coefficient of determination compared with the no-tuned model. There was no significant difference between the angles estimated by the tuned model activated by the electric pulse and muscle excitation. It indicates that neural activation could be replaced by the electric pulse to excite the limbs for desired angle. Therefore, the study presents a good way to evaluate the feasibility of Functional Electric Stimulation to suppress tremor.
\end{abstract}

Keywords: Tremor, musculoskeletal model, sEMG, electric pulse, function electric stimulation

\section{Introduction}

Tremor is an involuntary movement with a roughly sinusoidal profile [1]. The pathological tremor usually occurs in the wrist or elbow joint of patients suffering from Parkinson's disease and significantly weakens the daily activities, such as holding meal spoon or inserting the keys. Recently, Functional Electric Stimulation (FES) was intensively studied to restore the function of pathological extremities [2-5]. The exploration of the origin of tremor is fundamental to improving the treatments. Therefore, the musculoskeletal model of elbow joint involving mechanical properties and skeletal geometry [6] was developed to study tremor and to evaluate FES before acting on patients. Incidentally, the brachialis makes small contributions to the extension of elbow joint and its deep location blocks the accurate measurement of sEMG. Therefore, Biceps Brachii (BB) and Triceps Brachii (TB) are considered as the major muscles and Brachialis is neglected in this study.

Individuals have unique inherent anatomy and physiological parameters like the optimal fiber length

\footnotetext{
*Address for correspondence: Shengxin Wang, State Key Laboratory of Robotics and System, Harbin Institute of Technology, No. 2 Yikuang Street, Nan Gang District, Harbin 150080, China. Tel.:+86 0451 86413382; Fax: +86 04518641 3392; E-mail: hwsx89@hotmail.com.
} 
and maximum isometric force [6]. However, the values of these parameters cannot be directly measured in vivo, and are scaled from the literatures or measured from cadavers. These inappropriate values result in the developed model drastically differing from the corresponding subjects-specific model [6-8]. Furthermore, the developed model was sensitive to the characteristic of force-length and the maximum isometric force in rhythmic tasks [9]. Therefore, the muscle contraction parameters were tuned to calibrate the model and to minimize the discrepancy between the estimated and measured angles. Subsequently, the electric pulse (EP) modulated by sEMG instead of the muscle activation was used to excite the model. Finally, the difference between the measured angle and the angle estimated by the EP-driven model was tested by the coefficient of determination $\left(\mathrm{R}^{2}\right)$ and normalized root mean square error (NRMSE). Thus, the purpose of this study, to evaluate the feasibility of suppressing tremor by FES being used on the musculoskeletal model rather than patients, was realized.

\section{Methods}

\subsection{Derivation of muscle activation}

In this section, the derivation of the muscle activation is consisted of two sub-components: (1) the activation obtained from the sEMG of TB and BB by the linear envelope procedure (2) the activation acquired from $\mathrm{EP}$ that is modulated by $\mathrm{SEMG}$ of TB and $\mathrm{BB}$.

\subsection{1 sEMG-muscle activation dynamic}

The raw sEMG was normalized by the maximum voluntary contraction, fully wave rectified and filtered using a fourth order Butterworth low-pass filter with cut-off frequency $4 \mathrm{~Hz}$ [7]. The secondorder discrete linear recursive filter as Eq. (1) was employed to derive the muscle activation $(u(t))[6]$.

$$
\alpha e(t-d)-\beta_{1} u(t-1)-\beta_{2} u(t-2)=u(t)
$$

where $d$ is the electromechanical delay ranged between 10 and $100 \mathrm{~ms}$ [9]. The recursive coefficients, $\alpha, \beta_{1}$ and $\beta_{2}$, and the range are illustrated in Eqs. (2) and (3).

$$
\begin{gathered}
\beta_{1}=\gamma_{1}+\gamma_{2}, \beta_{2}=\gamma_{1} \times \gamma_{2},\left|\gamma_{1}\right|<1,\left|\gamma_{2}\right|<1 \\
\alpha-\beta_{1}-\beta_{2}=1
\end{gathered}
$$

Eq. (4), an exponential function, accounts for the relationship that the non-linear increases of muscle force at low level of sEMG and the nearly linear increase at higher level of sEMG [6]. $a(t)$ is the instantaneous muscle activation and $A$ is the nonlinear shape factor with physiologic constraint $[-3,0]$.

$$
a(\mathrm{t})=\left(e^{A u(\mathrm{t})}-1\right) /\left(e^{A}-1\right)
$$

\subsubsection{EP-muscle activation dynamic}

Rectangular pulse trains were usually selected in the application of FES. The pulse width was controlled to modulate the activation of the artificially stimulated muscle, while the pulse amplitude and frequency were pre-fixed. The muscle recruitment dynamic of motor units is expressed by a piecewise-linear function of pulse width shown below $[5,10]$. 


$$
a_{r}(d)=c_{1}\left\{\left(d-d_{\mathrm{thr}}\right) \arctan \left[\kappa_{\mathrm{thr}}\left(d-d_{\mathrm{thr}}\right)\right]-\left(d-d_{\text {sat }}\right) \arctan \left[\kappa_{\text {sat }}\left(d-d_{\text {sat }}\right)\right]\right\}+c_{2}
$$

where $d_{t h r}$ and $d_{\text {sat }}$ are the threshold and saturation values of pulse width, respectively. The curvatures of the recruitment curve is tuned by the constants, $\kappa_{\text {thr }}, \kappa_{\text {sat }}, c_{1}$ and $c_{2}$. The frequency characteristic of a single unit is shown as the function of stimulation frequency in Eq. $(6)[5,10]$.

$$
a_{f}(f)=(\alpha f)^{2} /\left(1+(\alpha f)^{2}\right)
$$

Calcium dynamics modeled by a first order differential equation as shown in Eq. (7) was used to explain the time delay between muscle activation and relaxation [4].

$$
d a / d t=\left(u^{2}-u a\right) / \tau_{a c}+(u-a) / \tau_{d a}
$$

where $\tau_{a c}$ and $\tau_{d a}$ are the activation time constant and de-activation time constant, respectively. $a$ is the muscle activation of a non-fatigue muscle, and $u=a_{f} a_{r}$.

During the movement of limbs, the muscle fibers were asynchronously activated by neural commands with a frequency of 6-8 Hz. However, the frequency was increased up to $20 \mathrm{~Hz}$ or higher when EP was used to stimulate the muscle fiber in a synchronous way. Therefore, the effects of muscle fatigue are considered and described by the first-order fitness function as follows $[4,5,10]$.

$$
\begin{gathered}
d f i t / d t=\left(f i t_{\min }-f i t\right) a \lambda(f) / T_{f a t}+(1-f i t)(1-a \lambda(f)) / T_{r e c} \\
\lambda(f)=1-\beta+\beta(0.01 f)^{2}
\end{gathered}
$$

where $T_{\text {fat }}$ and $T_{\text {rec }}$ denote the fatigue time constant and recovery time constant, respectively. The minimum fitness of muscle is denoted by $f_{i t} t_{\min }$ and $\beta$ represents the shaping factor. $\lambda$ accounts for the phenomenon that the muscle fatigue rate strongly depends on stimulation frequency. Finally, the muscle activation with fatigue was derived as shown in Eq. (10). The values of model parameters were scaled from literatures $[4,5,10]$.

$$
a_{m}(t)=a(t) f i t(t)
$$

\subsection{Muscle contraction dynamic}

Based on the Hill-type model, Zajac mathematically modeled the muscle-tendon unit as a contractile element in series with a tendon and in parallel with an elastic element [6]. Therefore, the muscle forces were calculated as the sum of the active force $\left(F_{a}{ }^{m}\right)$ corresponding to the muscle contractile element, and the passive force $\left(F_{p}{ }^{m}\right)$ responding to stretch and compression of the passive element as Eq. (11).

$$
F^{m}=F_{a}^{m}+F_{p}^{m}=\left(a(t) f_{a}\left(l_{m}\right) f_{a}\left(v_{m}\right)+f_{p}\left(l_{m}\right)\right) F_{\max } \cos \varphi
$$

where $F_{\max }$ represents maximum isometric muscle force at optimal fiber length $l_{m}{ }^{0} . \varphi$ is the penantion angle between the direction of tendons and the muscle fiber. $f_{a}\left(l_{m}\right)$ and $f_{a}\left(v_{m}\right)$ approximately define the 
normalized length-force and normalized velocity-force relationship, respectively. $f_{p}\left(l_{m}\right)$ is the normalized length-force in passive fiber.

To include more muscle contractile behavior, the instantaneous pennation angle $\varphi(t)$ was derived as the function of muscle fiber length and optimal pennation angle [11]. Additionally, the coupling between activation and optimal fiber length was also incorporated into the model [12].

\subsection{Musculoskeletal geometry}

Due to the constant distances between the elbow joint axis and the original, insertion points of $\mathrm{BB}$ and TB, the muscle length was derived from the variable component relative to the angle and the constant using trigonometry [13]. Next, the moment arm of each muscle was calculated readily from its length and joint angle by the tendon displacement method [14]. The muscle moment was then estimated by multiplying the muscle force and responding moment arm. In the sagittal plane, the angular acceleration of the joint was calculated by using joint forward dynamics as shown in Eq. (12).

$$
M+m g d \cos \theta=J d^{2} \theta / d t^{2}
$$

where $M$ is the net joint moment and $J$ is the moment of inertia. $m$ and $d$ indicate the sum mass of forearm and hand, and the distance between the mass center and joint axis, respectively. The parameters' values of musculoskeletal geometry were taken from literatures $[4,15]$.

The block diagram of the musculoskeletal model with the measured joint angle, and processed sEMG or EP as inputs is illustrated in Figure 1.

\subsection{Model calibration procedures}

Due to the unique inherent characteristic, the musculoskeletal model was firstly calibrated to obtain a generic set of model parameters for each subject. The more important muscle contraction parameters $l_{m}{ }^{0}, F_{m}{ }^{0}$ and $V_{m}{ }^{0}$ were selected as the adjustable parameters, with the activation dynamic variables being fixed [9]. In the literatures, the length of multiple heads was averaged as the optimal muscle fiber length and the peak isometric force was calculated by multiplying the cross-sectional area and

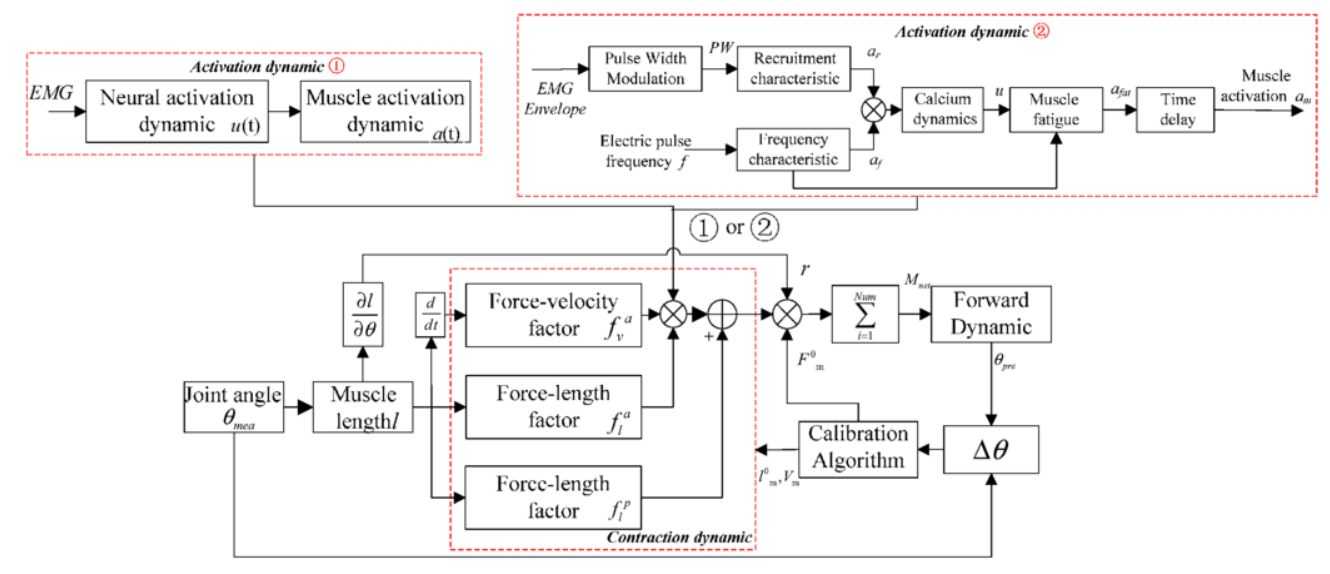

Fig. 1. Block diagram of the musculoskeletal model. 
the factor, $330 \mathrm{kPa}$. Finally, the average of the scaled values was considered as the initial values of the model parameters. In addition, $F_{m}{ }^{0}$ and $V_{m}{ }^{0}$ were allowed to vary between $50 \%$ and $200 \%$ of their initial values. $l_{m}{ }^{0}$ was constrained to change within $\pm 15 \%$ of its initial values [9].

The model parameters were tuned by the Reduced Gradient Method for minimizing the root mean square difference between the measured and predicted joint angle as shown in Eq. (13).

$$
J=\min \sum_{1}^{n}\left(\theta^{\text {pre }}-\theta^{m e s}\right)^{2}
$$

where $\theta^{\text {pre }}$ is the joint angle predicted by model and $\theta^{\text {mes }}$ is the measured joint angle

\section{Experiment and results}

\subsection{Data acquisition and process}

It is reported that tremor signals measured from mimicking healthy subject approximated patients' signals with similar frequency and higher amplitude [16, 17]. Therefore, four healthy subjects without a history of neuromuscular disorders were selected and introduced to the experimental protocol. The experiments were completed with the approval of the local ethics committee and were in accordance with the Declaration of Helsinki. Subjects were required to complete three different types of movement without any voluntary movement in sagittal plane: (1) the maximum voluntary contraction of TB and BB at the neutral position for three times; (2) the elbow flexion-extension movement with frequency of about $1 \mathrm{~Hz}$ and $5 \mathrm{~Hz}$ around the neutral position; The acquisition of each group lasted for roughly $20 \mathrm{~s}$ and subjects had a rest for $10 \mathrm{~min}$ between two groups of tasks to alleviate muscle fatigue.

The joint angle and sEMG of BB and TB were simultaneously measured by a Multimedia biofeedback and data acquisition system (Thought Technology Ltd, Canada) with sampling frequency of $2048 \mathrm{~Hz}$. The raw measured data was re-sampled by $128 \mathrm{~Hz}$ to decrease the calculation and then was processed offline by using Matlab (MathWorks, Inc) as expressed in section 2.1 to get the muscle activation. It should be noted that the positions and orientations of electrodes relative to the muscle usually changed during the limb's motion. Therefore, the sEMG could not be accurately measured and it was normalized by the maximum sEMG amplitude to decrease the effect of measurement error.

\subsection{Calibration of musculoskeletal model}

Before calibrating the model to subjects, the initial values of sensitive contraction parameters were

Table 1

Parameters and values of muscle model

\begin{tabular}{|c|c|c|c|c|c|c|c|c|c|c|c|c|}
\hline \multirow[t]{2}{*}{ Parameter } & Initial & 1 & 2 & 3 & 4 & \multirow{2}{*}{$\begin{array}{l}\text { Mean } \\
\text { (std) }\end{array}$} & Initial & 1 & 2 & 3 & 4 & \multirow{2}{*}{$\begin{array}{l}\text { Mean } \\
\text { (std) }\end{array}$} \\
\hline & value & Biceps & Brachii & & & & value & Tricep & Brachii & & & \\
\hline$F_{m}^{0}(N)$ & 449.2 & 418.7 & 409.1 & 412.3 & 425.2 & $416.3(7.13)$ & 944.3 & 603.5 & 563.3 & 526.7 & 542.3 & $\begin{array}{l}558.9 \\
(33.3)\end{array}$ \\
\hline$L_{m}^{0}(\mathrm{~cm})$ & 151.3 & 152.6 & 158.3 & 149.6 & 153.6 & $\begin{array}{l}153.5 \\
(3.61)\end{array}$ & 111.3 & 117.4 & 121.4 & 112.6 & 118.3 & $\begin{array}{l}117.4 \\
(3.64)\end{array}$ \\
\hline$V_{m}^{0}(m / s)$ & 0.68 & 0.76 & 0.89 & 0.73 & 0.75 & $\begin{array}{l}0.78 \\
(0.07)\end{array}$ & 0.42 & 0.48 & 0.69 & 0.52 & 0.58 & $\begin{array}{l}0.57 \\
(0.09)\end{array}$ \\
\hline
\end{tabular}


taken from literatures and were list in Table $1[3,14]$. Based on the optimizing approach, the calibrated values of parameters of each subject were averaged across 3 trails as shown in Table 1. By comparing the average tuned values and initial values, the difference indicated the necessity of model calibration.

The initial/ tuned values of the model parameters were selected to estimate the joint angle, and the results were shown in Figure 2. The output of the no-tuned model did not capture the measured joint angle. Nevertheless, an agreement in shape and magnitude between the output of the calibrated model and the measured angle were observed. The average $\mathrm{R}^{2}$ and NRMSE with standard deviations in parentheses across rhythmical tasks and subjects were calculated and tabulated in Table 2. The values in the column were calculated by averaging three trails of each subject. By comparing the average values between the no-tuned and tuned model, an increase by 0.05 in $\mathrm{R}^{2}$, and a decrease by $10.76 \%$ in NRMSE are observed. This improvement further demonstrates that the initial values of the parameters obtained from the literature were not appropriate for the subjects.

\subsection{Activation of musculoskeletal system by EP}

Once the musculoskeletal model was calibrated, EP provided by the stimulator was used to activate the model. In this study, the amplitude and frequency of EP were fixed at $20 \mathrm{~Hz}$ and $30 \mathrm{~mA}$, respectively. The pulse width was modulated by the sinusoidal Pulse Width Modulation (PWM) technology being used on the envelope of sEMG. Compared with the activation delivered from neural

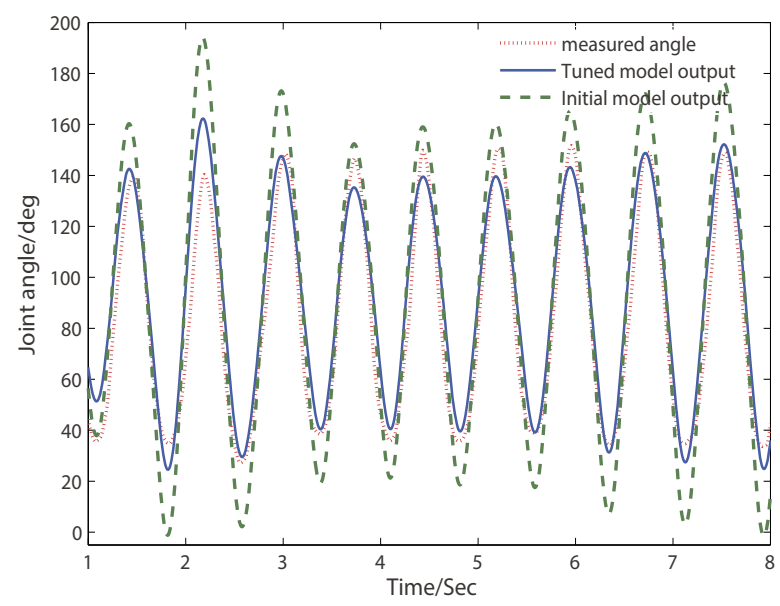

Fig. 2. Measured and modeled elbow joint angle for a representative trial (subject 2).

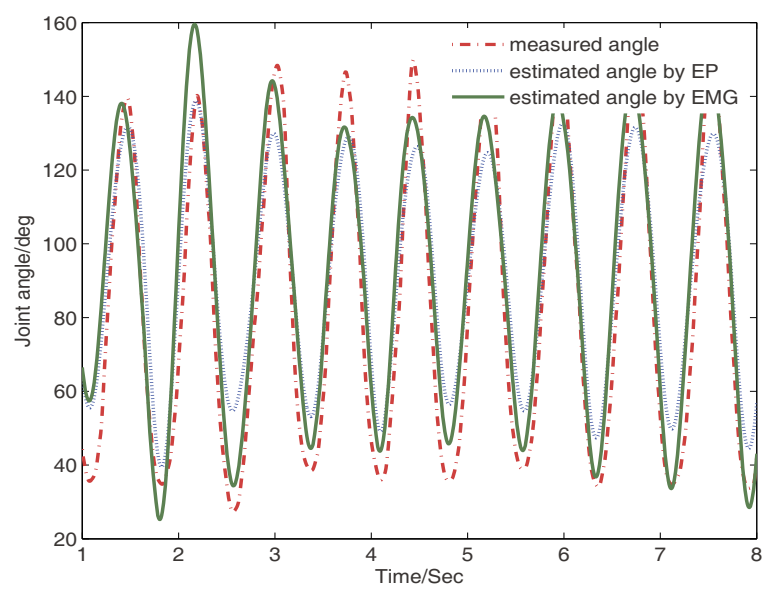

Fig. 3. Measured, stimulated and modeled joint angle for a representative trial (subject 2).

Table 2

Estimation of No-calibrated and Calibrated model activated by sEMG and EP, respectively

\begin{tabular}{lllllll}
\hline \multirow{2}{*}{ Subject } & \multicolumn{2}{l}{ No-Calibrated model } & \multicolumn{2}{l}{ Calibrated model(sEMG) } & \multicolumn{2}{l}{ Calibrated model(EP) } \\
\cline { 2 - 7 } & $\mathrm{R}^{2}$ & NRMSE & $\mathrm{R}^{2}$ & NRMSE & $\mathrm{R}^{2}$ & NRMSE \\
\hline 1 & $0.87(0.03)$ & $32.94(2.23)$ & $0.92(0.01)$ & $21.86(0.91)$ & $0.90(0.01)$ & $23.59(1.63)$ \\
2 & $0.85(0.01)$ & $36.38(1.79)$ & $0.91(0.01)$ & $26.38(1.67)$ & $0.89(0.02)$ & $28.57(4.21)$ \\
3 & $0.86(0.03)$ & $35.12(2.02)$ & $0.91(0.02)$ & $24.06(1.84)$ & $0.89(0.01)$ & $26.49(2.32)$ \\
4 & $0.86(0.04)$ & $34.78(1.41)$ & $0.91(0.01)$ & $23.90(1.23)$ & $0.88(0.01)$ & $25.67(2.13)$ \\
Mean(std) & $0.86(0.01)$ & $34.81(0.85)$ & $0.91(0.01)$ & $24.05(0.94)$ & $0.89(0.01)$ & $26.08(1.03)$ \\
\hline \multicolumn{7}{c}{ Note: $\mathrm{R}^{2:}$ coefficient of determination; NRMSE: normalized root mean square error. }
\end{tabular}


system, the effect of the external electric stimulation was greatly diminished by the muscle fatigue, without real-time compensation by the neural system. Therefore, a gain was derived by the muscle fatigue and was introduced to correct the EP for expected outputs.

The angle predicted by the model, together with the measured angle, was shown in Figure 3 while $\mathrm{R}^{2}$ and NRMSE across three tasks were listed in Table 2. It was found that the estimated angle followed the measured angle closely, with a smaller $\mathrm{R}^{2}$ by 0.02 , and a larger NRMSE by $2.03 \%$. Therefore, the results indicated that EP could be employed to activate the model instead of muscle activation to obtain the desired angle in the future.

\section{Discussion}

In the literature, EMG-driven musculoskeletal models are widely employed to estimate muscle forces/joint moment $[4,6,7,9]$. However, ethical considerations prevent the in vivo measurement of muscle forces, and the indirectly calculated joint moment readily impacted by the perturbation. Furthermore, the values of parameters from literatures are inconsistent with subject-specific values [8]. The difference between the no-tuned model and tuned model is list in Table 1 and gives further confidence that subject-specific parameters more accurately represent the anatomy and physiology of muscle system. Therefore, the discrepancy between the estimated and directly measured angle was used to calibrate the musculoskeletal model to individuals over limited sets of data [9]. To save time, the sensitivity of parameters was evaluated to divide the parameters into global and muscle-specific groups.

When FES was used to suppress pathological tremor, EP generated by the stimulator was introduced instead of neural activation to excite the muscle $[5,10]$. To explore the performance of FES, FES was firstly evaluated based on the calibrated model before being used on patients. Next, the width of EP was modulated by the envelope of sEMG with sinusoidal PWM technology, and its frequency and amplitude are fixed. The experimental results shown in Table 2 illustrate that the estimated angle closely follows the measured angle. Additionally, the angle estimated by EP approximates the output of model with the processed EMG as activation. Figure 3 visually provides insight into the inference that EP could be employed to activate the muscle model, and subsequently, the patients' muscle. However, the model will be improved by including more muscle contractile behavior and peripheral neural system to increase the prediction accuracy and model generalization in future studies.

\section{Conclusion}

In this paper, a generic musculoskeletal model was developed and later was calibrated to subjects. The activation derived from EP, instead of muscle activation, successfully excited the muscle model and obtained the desirable joint angle. This exploration lays a basis for simulating the antagonistic muscles system to study tremor and to evaluate the performance of FES modulated by processed sEMG before being used on patients.

\section{Acknowledgments}

The study is funded and supported by "National Natural Science Foundation" (Grant No. 60975067) 
and "National Natural Science Foundation" (Grant No. 61473104).

\section{References}

[1] R.J. Elble, W.C. Koller and Tremor, MD Dissertation, Johns Hopkins University, Baltimore, 1990.

[2] J.M. Belda-Lois, et al., Controllable mechanical tremor reduction, Assessment of two orthoses, Journal of Technology and Disability 19 (2007), 169-178.

[3] Arthur Prochazka, Josef Elek et al., Attenuation of pathological tremors by functional electrical stimulation I, Method, Annals of Biomedical Engineering 20 (1992), 205-224.

[4] Dingguo Zhang, et al., Neural oscillator based control for pathological tremor suppression via functional electrical stimulation, Control Engineering Practice 19 (2011), 74-88.

[5] Robert Riener and Thomas Fuhr, Patient-driven control of FES-supported standing up: A simulation study, IEEE Transactions on Rehabilitation Engineering 6 (1998), 113-126.

[6] F.E. Zajac, Muscle and tendon, Properties, models, scaling, and application to biomechanics and motor control, Critical Reviews in Biomedical Engineering 17 (1989), 359-411.

[7] David G. Lloyd and Thor F. Besier, A sEMG-driven musculoskeletal model to estimate muscle forces and knee joint moments in vivo, Journal of Biomechanics 36 (2003), 765-776.

[8] Carol Y. Scovil and Janet L. Ronsky, Sensitivity of a Hill-based muscle model to perturbations in model parameters, Journal of Biomechanics 39 (2006), 2055-2063.

[9] Qi Shao, Daniel N. Bassett, Kurt Manal and T.S. Buchanan, An EMG-driven model to estimate muscle forces and joint moments in stroke patients, Computer in Biology and Medicine 39 (2009), 1083-1088.

[10]R. Riener and J. Quintern, A physiologically based model of muscle activation verified by electrical stimulation, Bioelectrochemistry and Bioenergetics 43 (1997), 257-264.

[11] S.H. Scott and D.A. Winter, A comparison of three muscle pennation assumptions and their effect on isometric and isotonic force, Journal of Biomechanics 24 (1994), 163-167.

[12] P.A. Huijing, Important experimental factors for skeletal muscle modelling: Non-linear changes of muscle length force characteristics as a function of degree of activity, European Journal of Morphology 34 (1996), 47-54.

[13] P. Pigeon, et al., Moment arms and lengths of human upper limb muscles as functions of joint angles, Journal of Biomechanics. 29 (1996), 1365-1370.

[14] K.N. An, et al., Determination of muscle orientations and moment arms, Journal of Biomechanics Engineering 106 (1984), 280-282.

[15] Paolo de Leva, Adjustments to Zatsiorsky-seluyanov's segment inertia parameters, Journal of Biomechanics 29 (1996), $1223-1230$.

[16] T.S. Buchanan, S.L. Delp and J.A. Solbeck, Muscular resistance to varus and valgus loads at the elbow, Journal of Biomechanics Engineering 120 (1998), 634-639.

[17] Bettina Pollok, Joachim Gross, Martin Dirks, et al., The cerebral oscillatory network of voluntary tremor, Journal of Physiology 3 (2003), 871-878. 